\title{
An Inverse Problem for a Two-Dimensional Time-Fractional Sideways Heat Equation
}

\author{
Songshu Liu ${ }^{1}{ }^{1}$ and Lixin Feng $\mathbb{D}^{2}$ \\ ${ }^{1}$ School of Mathematics and Statistics, Northeastern University at Qinhuangdao, Qinhuangdao 066004, China \\ ${ }^{2}$ School of Mathematical Sciences, Heilongjiang University, Harbin 150080, China \\ Correspondence should be addressed to Lixin Feng; fenglixin@hlju.edu.cn
}

Received 22 November 2019; Accepted 3 February 2020; Published 10 March 2020

Academic Editor: Pietro Bia

Copyright ( $\odot 2020$ Songshu Liu and Lixin Feng. This is an open access article distributed under the Creative Commons Attribution License, which permits unrestricted use, distribution, and reproduction in any medium, provided the original work is properly cited.

In this paper, we consider a two-dimensional (2D) time-fractional inverse diffusion problem which is severely ill-posed; i.e., the solution (if it exists) does not depend continuously on the data. A modified kernel method is presented for approximating the solution of this problem, and the convergence estimates are obtained based on both a priori choice and a posteriori choice of regularization parameters. The numerical examples illustrate the behavior of the proposed method.

\section{Introduction}

In the past decades, fractional differential equations have attracted wide attention. Various models using fractional partial differential equations have been successfully applied to describe a range of problems in mechanical engineering [1], viscoelasticity [2], electron transport [3], dissipation [4], heat conduction [5, 6], and high-frequency financial data [7].

The time-fractional diffusion equation is deduced by replacing the standard time derivative with a time-fractional derivative and can be used to describe the superdiffusion and subdiffusion phenomena [8-13]. The direct problems, i.e., initial value problems and initial boundary value problems, for the time-fractional diffusion equation have been studied extensively in recent years. For instance, a well-posedness analysis [14-18] and numerical methods and simulations [19-24] are typical examples of this kind of analysis.

However, in some practical situations, part of boundary data, or initial data, or diffusion coefficient, or source term, or the order of fractional derivative may not be given and we want to find them by additional measured data which will yield some inverse problems of the fractional diffusion equation. The early papers on inverse problems of timefractional diffusion equation were provided by Murio in $[25,26]$ for solving the time-fractional diffusion equation by mollification methods. Cheng et al. [27] gave the uniqueness in determining the parameter $\alpha$ and $p(x)$ by means of observation data at one end point. Liu and Yamamoto [28] considered a backward problem in time for a time-fractional partial diffusion equation in one-dimensional case. Zhang and $\mathrm{Xu}$ [29] studied an inverse source problem in the timefractional diffusion equation and proved uniqueness for identifying a space-dependent source term by using analytic continuation and Laplace transform. Zheng and Wei [30] applied a new regularization method to solve an inverse problem for a time-fractional diffusion equation in a onedimensional semi-infinite domain. Jin and Rundell [31] studied an inverse problem of recovering a spatially varying potential term in the $1 \mathrm{D}$ time-fractional diffusion equation from the flux measurements and proposed a quasi-Newtontype reconstruction algorithm. Recently, Wei and Wang [32] proposed a modified quasi-boundary value method for an inverse source problem of the time-fractional diffusion equation. Wei et al. [33] identified a time-dependent source term in a multidimensional time-fractional diffusion equation from boundary Cauchy data.

As for time-fractional inverse diffusion problem for onedimensional (1D) models, theoretical concepts and computational implementation have been discussed by many authors, and a number of solution methods have been proposed, i.e., spectral regularization method [34], iteration regularization method [35], optimal regularization method 
[36], and a new dynamic method [37]. However, for the twodimensional time-fractional inverse diffusion problem, few results are available. Xiong et al. [38] gave a conditional stability estimate for the inverse heat conduction problem in the $2 \mathrm{D}$ time-fractional heat equation and studied a dynamic spectral regularization method with numerical testification. However, error estimates were given in [38] by only choosing the regularization parameter by a priori choice rule.

In the present paper, we will consider the $2 \mathrm{D}$ time-fractional inverse diffusion problem by using a modified kernel method and a posteriori parameter choice rule is given. For a priori choice and a posteriori choice of the regularization parameter, we obtain the convergence estimates, respectively.

The rest of this paper is organized as follows. In Section 2 , we describe the $2 \mathrm{D}$ time-fractional inverse diffusion problem and give an analysis on the ill-posedness of this problem. In Section 3, we propose a modified kernel method and prove the convergence estimates under a priori and a posteriori parameter choice rule, respectively. Section 4 is the numerical aspect of the proposed method. Finally, we give a brief conclusion in Section 5 .

\section{Description of the 2D Time-Fractional Inverse Diffusion Problem}

We consider the following 2D time-fractional inverse diffusion problem:

$$
\begin{array}{r}
\frac{\partial^{\alpha} u}{\partial t^{\alpha}}-u_{x x}-u_{y y}=0, \quad x>0, y>0, t>0, \\
u(1, y, t)=g(y, t), \quad y \geq 0, t \geq 0,
\end{array}
$$

with the corresponding measured data function $g^{\delta}(y, t)$ and initial and boundary conditions

$$
\begin{gathered}
u(x, y, 0)=0, \quad x>0, \quad y \geq 0, \\
u(x, 0, t)=0, \quad x>0, t \geq 0, \\
u_{y}(x, 0, t)=0, \quad x>0, t \geq 0, \\
\left.u(x, y, t)\right|_{x \rightarrow \infty} \text { bounded, } \quad y>0, t>0,
\end{gathered}
$$

where the time-fractional derivative $\left(\partial^{\alpha} u / \partial t^{\alpha}\right)$ is the Caputo fractional derivative of order $\alpha(0<\alpha \leq 1)$ defined by (see [39])

$$
\begin{aligned}
& \frac{\partial^{\alpha} u}{\partial t^{\alpha}}(x, y, t)=\frac{1}{\Gamma(1-\alpha)} \int_{0}^{t} \frac{\partial u(x, y, s)}{\partial s} \frac{\mathrm{d} s}{(t-s)^{\alpha}}, \quad 0<\alpha<1, \\
& \frac{\partial^{\alpha} u}{\partial t^{\alpha}}(x, y, t)=\frac{\partial u(x, y, t)}{\partial t}, \quad \alpha=1 .
\end{aligned}
$$

Here, we wish to determine the temperature $u(x, y, t)$ for $0 \leq x<1$ from the temperature measurements $g^{\delta}(y, t)$.

In order to apply the Fourier transform techniques, we extend the functions $u(x, \cdot, \cdot), g(\cdot, \cdot)$, and $g^{\delta}(\cdot, \cdot)$ to the whole plane $-\infty<y<+\infty$ and $-\infty<t<+\infty$ by setting the functions to be zero for $y<0$ or $t<0$. We assume that these functions are in $L^{2}\left(\mathbb{R}^{2}\right)$ and use the corresponding $L^{2}$-norm, defined as follows:

$$
\|g\|=\left(\int_{\mathbb{R}^{2}}|g(y, t)|^{2} \mathrm{~d} y \mathrm{~d} t\right)^{1 / 2} .
$$

We also assume that the measured data function $g^{\delta}(y, t)$ satisfies

$$
\left\|g^{\delta}-g\right\| \leq \delta,
$$

where the constant $\delta>0$ represents a bound on the measurement error.

$$
\begin{aligned}
& \text { Let } \\
& \hat{g}(\xi, \eta)=\frac{1}{2 \pi} \int_{\mathbb{R}^{2}} g(y, t) e^{-i(\xi y+\eta t)} \mathrm{d} y \mathrm{~d} t, \quad \xi, \eta \in \mathbb{R},
\end{aligned}
$$

be the Fourier transform of a function $g(y, t)$. Taking the transform to equations (1)-(6) with respect to $y$ and $t$, we can get the solution of equations (1)-(6) in the frequency domain (see [38]):

$$
\widehat{u}(x, \xi, \eta)=e^{(1-x) \sqrt{(i \eta)^{\alpha}+\xi^{2}}} \hat{g}(\xi, \eta),
$$

where

$$
(i \eta)^{\alpha}=|\eta|^{\alpha}\left(\cos \left(\frac{\alpha \pi}{2}\right)+i \operatorname{sign}(\eta) \sin \left(\frac{\alpha \pi}{2}\right)\right) .
$$

Denote $\gamma$ as follows:

$$
\gamma:=\sqrt{(i \eta)^{\alpha}+\xi^{2}}=A+i B,
$$

where

$$
\begin{aligned}
& A:=\sqrt{\frac{\sqrt{|\eta|^{2 \alpha} \sin ^{2}(\alpha \pi / 2)+\left(\xi^{2}+|\eta|^{\alpha} \cos (\alpha \pi / 2)\right)^{2}}+\left(\xi^{2}+|\eta|^{\alpha} \cos (\alpha \pi / 2)\right)}{2}}, \\
& B:=\operatorname{sign}\left(\operatorname{sign}(\eta)|\eta|^{\alpha} \sin \left(\frac{\alpha \pi}{2}\right)\right)
\end{aligned}
$$

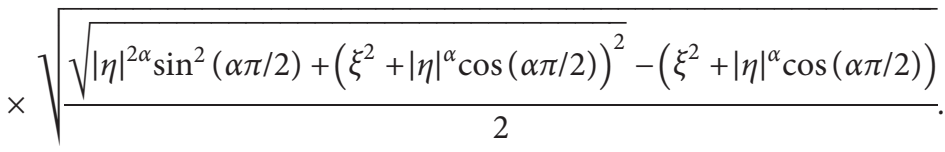


For the above problem, since $\left|e^{(1-x) \sqrt{(i \eta)^{\alpha}+\xi^{2}}}\right|$ is unbounded with respect to variables $\xi$ and $\eta$ for fixed $0 \leq x<1$, the small error in the high-frequency components will be amplified. Therefore, the 2D time-fractional inverse diffusion problem is severely ill-posed. To solve the $2 \mathrm{D}$ time-fractional inverse diffusion problem, a natural way to stabilize the problem is eliminate the high frequencies or to replace the "kernel" $e^{(1-x) \sqrt{(i \eta)^{\alpha}+\xi^{2}}}$ by a bounded approximation.

\section{A Modified Method and Convergence Estimates}

In this section, we will give a modified "kernel" method and obtain the convergence estimates. The regularization solution is given by

$$
\widehat{u}_{\beta}^{\delta}(x, \xi, \eta)=k_{\beta}(x, \xi, \eta) \widehat{g}^{\delta}(\xi, \eta)
$$

where

$$
k_{\beta}(x, \xi, \eta)= \begin{cases}e^{(1-x)(A+i B)}, & e^{(1-x) A} \leq \beta(x), \\ \beta(x) e^{i(1-x) B}, & e^{(1-x) A}>\beta(x),\end{cases}
$$

where $\beta(x)>1$ is the regularization parameter.

To obtain the convergence estimates between the regularization solution and the exact solution, we need to assume a priori bound:

$$
\|u(0, \cdot, \cdot)\|_{p} \leq E, \quad p>0,
$$

where $E>0$ is a constant and $\|\cdot\|_{p}$ denotes the norm in Sobolov space $H^{p}\left(\mathbb{R}^{2}\right)$ defined by

$$
\|u(x, \cdot, \cdot)\|_{p}:=\left(\int_{\mathbb{R}^{2}}\left(1+\xi^{2}+\eta^{2}\right)^{p}|\widehat{u}(0, \cdot, \cdot)|^{2} \mathrm{~d} \xi \mathrm{d} \eta\right)^{1 / 2} .
$$

Remark 1. Obviously, when $p=0$, we can know that $H^{p}\left(\mathbb{R}^{2}\right)=H^{0}\left(\mathbb{R}^{2}\right)=L^{2}\left(\mathbb{R}^{2}\right)$ and formula (17) is bounded in the $L^{2}\left(\mathbb{R}^{2}\right)$-norm.

3.1. A Priori Parameter Choice. In the following, we give the convergence estimate for $\left\|u_{\beta}^{\delta}(x, \cdot, \cdot)-u(x, \cdot, \cdot)\right\|$ by using a priori choice rule for the regularization parameter.

Theorem 1. Suppose that $u_{\beta}^{\delta}(x, y, t)$ is the regularization solution with noisy data $g^{\delta}(y, t)$ and that $u(x, y, t)$ is the exact solution with the exact data $g(y, t)$. Let assumption (9) be satisfied and let $\|u(0, \cdot, \cdot)\| \leq E$. If we choose

$$
\beta(x)=x\left(\frac{\delta}{E}\right)^{x-1},
$$

then for every $x \in(0,1)$, we obtain the error estimate

$$
\left\|u_{\beta}^{\delta}(x, \cdot, \cdot)-u(x, \cdot, \cdot)\right\| \leq \delta^{x} E^{1-x} .
$$

Proof. Due to Parseval's identity and triangle inequality, we can obtain

$$
\begin{aligned}
\| u_{\beta}^{\delta} & (x, \cdot, \cdot)-u(x, \cdot, \cdot) \| \\
= & \left\|\widehat{u}_{\beta}^{\delta}(x, \cdot, \cdot)-\widehat{u}(x, \cdot, \cdot)\right\| \\
= & \left\|k_{\beta}(x, \xi, \eta) \widehat{g}^{\delta}-e^{(1-x)(A+i B)} \hat{g}\right\| \\
= & \| k_{\beta}(x, \xi, \eta) \widehat{g}^{\delta}-k_{\beta}(x, \xi, \eta) \widehat{g}+k_{\beta}(x, \xi, \eta) \widehat{g} \\
& -e^{(1-x)(A+i B)} \hat{g} \| \\
\leq & \left\|k_{\beta}(x, \xi, \eta) \hat{g}^{\delta}-k_{\beta}(x, \xi, \eta) \hat{g}\right\|+\| k_{\beta}(x, \xi, \eta) \widehat{g} \\
& -e^{(1-x)(A+i B)} \hat{g} \| \\
= & I_{1}+I_{2} .
\end{aligned}
$$

For $I_{1}$, we have

$I_{1}=\left\|k_{\beta}(x, \xi, \eta) \hat{g}^{\delta}-k_{\beta}(x, \xi, \eta) \hat{g}\right\| \leq \delta \sup _{\xi, \eta \in \mathbb{R}}\left|k_{\beta}(x, \xi, \eta)\right| \leq \delta \beta(x)$.

From (11), we know

$$
\widehat{g}(\xi, \eta)=e^{-(A+i B)} \widehat{u}(0, \xi, \eta) .
$$

We now estimate $I_{2}$; note that from (23),

$$
\begin{aligned}
I_{2} & =\left\|k_{\beta}(x, \xi, \eta) \widehat{g}-e^{(1-x)(A+i B)} \hat{g}\right\| \\
& =\left\|\frac{e^{(1-x)(A+i B)}-k_{\beta}(x, \xi, \eta)}{e^{(A+i B)}} \widehat{u}(0, \xi, \eta)\right\| \\
& =\left\|\frac{e^{(1-x)(A+i B)}-\min \left\{e^{(1-x) A}, \beta(x)\right\} e^{(1-x) i B}}{e^{(A+i B)}} \widehat{u}(0, \xi, \eta)\right\| \\
& =\left\|\frac{\left(e^{(1-x) A}-\min \left\{e^{(1-x) A}, \beta(x)\right\}\right) e^{(1-x) i B}}{e^{(A+i B)}} \widehat{u}(0, \xi, \eta)\right\| \\
& \leq \sup _{\xi, \eta \in \mathbb{R}}\left|\frac{\left(e^{(1-x) A}-\min \left\{e^{(1-x) A}, \beta(x)\right\}\right) e^{(1-x) i B}}{e^{(A+i B)}}\right| E \\
& \leq E \sup _{\xi, \eta \in \mathbb{R}, e^{(1-x) A}>\beta(x)} \frac{e^{(1-x) A}-\beta(x)}{e^{A}} .
\end{aligned}
$$

Let

$$
\varphi(A)=\frac{e^{(1-x) A}-\beta(x)}{e^{A}} .
$$

Differentiating $\varphi(A)$ and setting the derivative equal to zero, we find that

$$
A^{*}=\frac{1}{1-x} \ln \left(\frac{1}{x} \beta(x)\right),
$$

is the maximum value point of the function $\varphi(A)$. Therefore, we know

$$
I_{2} \leq \frac{((1 / x)-1) \beta(x)}{((1 / x) \beta(x))^{1 /(1-x)}} E .
$$

Combining (19), (21), (22), and (27), we have the Hölder-type error estimate (20). 
Remark 2. The error estimate in Theorem 1 does not give any useful information on the continuous dependence of the solution at $x=0$. To retain the continuous dependence of the solution at $x=0$, one has to introduce a stronger a priori assumption.

Theorem 2. Suppose that $u_{\beta}^{\delta}(0, y, t)$ is the regularization solution with noisy data $g^{\delta}(y, t)$ and that $u(0, y, t)$ is the exact solution with the exact data $g(y, t)$ at $x=0$. Let assumption (9) be satisfied and let $\|u(0, \cdot, \cdot)\|_{p} \leq E(p>0)$. If we choose

$$
\beta=\left(\frac{\delta}{E}\right)^{-(1 / 2)},
$$

then for $x=0$, we obtain the error estimate

$$
\left\|u_{\beta}^{\delta}(0, y, t)-u(0, y, t)\right\| \leq \delta^{1 / 2} E^{1 / 2}+E\left(\frac{1}{4} \ln \frac{E}{\delta}\right)^{-p} .
$$

Proof. Due to Parseval's identity and the triangle inequality, we have

$$
\begin{aligned}
& \left\|u_{\beta}^{\delta}(0, y, t)-u(0, y, t)\right\| \\
& \quad=\left\|\hat{u}_{\beta}^{\delta}(0, \cdot, \cdot)-\widehat{u}(0, \cdot \cdot)\right\| \\
& \quad=\left\|k_{\beta}(0, \xi, \eta) \widehat{g}^{\delta}-e^{(A+i B)} \widehat{g}\right\| \\
& \quad=\left\|k_{\beta}(0, \xi, \eta) \widehat{g}^{\delta}-k_{\beta}(0, \xi, \eta) \widehat{g}+k_{\beta}(0, \xi, \eta) \widehat{g}-e^{(A+i B)} \hat{g}\right\| \\
& \quad \leq\left\|k_{\beta}(0, \xi, \eta) \widehat{g}^{\delta}-k_{\beta}(0, \xi, \eta) \hat{g}\right\|+\left\|k_{\beta}(0, \xi, \eta) \widehat{g}-e^{(A+i B)} \hat{g}\right\| \\
& \quad=I_{3}+I_{4},
\end{aligned}
$$

where

$$
\begin{gathered}
I_{3}=\left\|k_{\beta}(0, \xi, \eta) \hat{g}^{\delta}-k_{\beta}(0, \xi, \eta) \hat{g}\right\| \\
=\left\|k_{\beta}(0, \xi, \eta)\left(g^{\delta}-g\right)\right\| \\
\leq \delta \sup _{\xi, \eta \in \mathbb{R}}\left|k_{\beta}(0, \xi, \eta)\right| \\
\leq \delta \beta, \\
I_{4}=\left\|k_{\beta}(0, \xi, \eta) \widehat{g}-e^{(A+i B)} \hat{g}\right\| \\
=\left\|\frac{e^{((A+i B)}-\min \left\{e^{A}, \beta\right\} e^{i B}}{e^{(A+i B)}} \widehat{u}(0, \xi, \eta)\right\| \\
=\left\|\frac{\left(e^{A}-\min \left\{e^{A}, \beta\right\}\right) e^{i B}}{\left(1+\xi^{2}+\eta^{2}\right)^{p / 2} e^{(A+i B)}}\left(1+\xi^{2}+\eta^{2}\right)^{p / 2} \widehat{u}(0, \xi, \eta)\right\| \\
\leq \sup _{\xi, \eta \in \mathbb{R}}\left|\frac{\left(e^{A}-\min \left\{e^{A}, \beta\right\}\right) e^{i B}}{\left(1+\xi^{2}+\eta^{2}\right)^{p / 2} e^{(A+i B)}}\right| E \\
\leq E \sup _{\xi, \eta \in \mathbb{R}, e^{A}>\beta} \frac{e^{A}-\beta}{\left(1+\xi^{2}+\eta^{2}\right)^{p / 2} e^{A}} .
\end{gathered}
$$
get

Using the fact $|\gamma|=\left|\sqrt{(i \eta)^{\alpha}+\xi^{2}}\right| \leq|\eta|^{\alpha / 2}+|\xi|$, we can

$$
A \leq \sqrt{A^{2}+B^{2}}=|\gamma| \leq|\eta|^{\alpha / 2}+|\xi| \text {, i.e., } A \leq|\eta|^{\alpha / 2}+|\xi| .
$$

Note that also $\beta<e^{A}$, and we have $A>\ln \beta$; therefore, we can derive

$$
\ln \beta \leq|\eta|^{\alpha / 2}+|\xi|
$$

If $|\xi|>|\eta|^{\alpha / 2} \geq 0$, we know that

$$
\ln \beta \leq|\eta|^{\alpha / 2}+|\xi| \leq 2|\xi|,
$$

i.e., $|\xi|>(1 / 2) \ln \beta$. Therefore,

$$
\left(1+\xi^{2}+\eta^{2}\right)^{-(p / 2)} \leq\left(\xi^{2}+\eta^{2}\right)^{-(p / 2)}<|\xi|^{-p}<\left(\frac{1}{2} \ln \beta\right)^{-p} .
$$

If $|\eta|^{\alpha / 2} \geq|\xi| \geq 0$, we have

$$
\ln \beta \leq|\eta|^{\alpha / 2}+|\xi| \leq 2|\eta|^{\alpha / 2},
$$

i.e., $|\eta| \geq((1 / 2) \ln \beta)^{2 / \alpha}$. Therefore,

$$
\left(1+\xi^{2}+\eta^{2}\right)^{-(p / 2)} \leq\left(\xi^{2}+\eta^{2}\right)^{-(p / 2)} \leq|\eta|^{-p}<\left(\frac{1}{2} \ln \beta\right)^{-((2 p) / \alpha)} .
$$

Combining (32), (36), and (38), we can obtain

$$
I_{4} \leq E \sup _{\xi, \eta \in \mathbb{R}, e^{A}>\beta} \frac{1}{\left(1+\xi^{2}+\eta^{2}\right)^{p / 2}} \leq E \max
$$

$$
\cdot\left\{\left(\frac{1}{2} \ln \beta\right)^{-p},\left(\frac{1}{2} \ln \beta\right)^{-((2 p) / \alpha)}\right\} \text {. }
$$

Combining (28), (30), (31), and (39), we can obtain

$$
\begin{aligned}
\left\|u_{\beta}^{\delta}(0, y, t)-u(0, y, t)\right\| \leq & I_{3}+I_{4} \\
\leq & \delta^{1 / 2} E^{1 / 2}+E \max \left\{\left(\frac{1}{4} \ln \frac{E}{\delta}\right)^{-p},\right. \\
& \left.\left(\frac{1}{4} \ln \frac{E}{\delta}\right)^{-((2 p) / \alpha)}\right\} \\
& =\delta^{1 / 2} E^{1 / 2}+E\left(\frac{1}{4} \ln \frac{E}{\delta}\right)^{-p} .
\end{aligned}
$$

3.2. A Posteriori Parameter Choice. In this section, we first give the following lemma.

Lemma 1 (see [40]). Let the function $f(\lambda):(0, a] \longrightarrow \mathbb{R}$ be given by 


$$
f(\lambda)=\lambda^{b}\left[d \ln \frac{1}{\lambda}\right]^{-c}
$$

with a constant $c \in \mathbb{R}$ and positive constants $a<1, b$, and $d$; then, for the inverse function, we have

$$
f^{-1}(\lambda)=\lambda^{1 / b}\left[\frac{d}{b} \ln \frac{1}{\lambda}\right]^{c / b}(1+o(1)), \quad \text { for } \lambda \longrightarrow 0 .
$$

In this following, we give the convergence estimate for $\left\|u_{\beta}^{\delta}(x, \cdot, \cdot)-u(x, \cdot, \cdot)\right\|$ by using a posteriori choice rule for the regularization parameter, i.e., Morozov's discrepancy principle.

According to Morozov's discrepancy principle [41], we adopt the regularization parameter $\beta$ as the solution of the following equation:

$$
\left\|e^{-(A+i B)(1-x)} k_{\beta}(x, \xi, \eta) \widehat{g}^{\delta}-\widehat{g}^{\delta}\right\|=\tau \delta,
$$

where $\tau>1$ is a constant. Denote

$$
T_{\beta}=e^{-(A+i B)(1-x)} k_{\beta}(x, \xi, \eta)-1 .
$$

Here and in the following, $\beta$ is a function of the variable $x$ and sometimes the function $\beta(x)$ is also used.

Lemma 2. Let $\rho(\beta):=\left\|e^{-(A+i B)(1-x)} k_{\beta}(x, \xi, \eta) \widehat{g}^{\delta}-\widehat{g}^{\delta}\right\|$, and then, the following results hold:
(a) $\rho(\beta)$ is a continuous function
(b) $\lim _{\beta \longrightarrow 1} \rho(\beta)=\left\|\left(e^{-A(1-x)}-1\right) \widehat{g}^{\delta}(\xi, \eta)\right\|$
(c) $\lim _{\beta \longrightarrow+\infty} \rho(\beta)=0$
(d) $\rho(\beta)$ is a strictly decreasing function for $\beta \in(1,+\infty)$

The proof is obvious, and we omit it here.

Remark 3. According to Lemma 2, we find that, if $0<\tau \delta<\left(e^{-A(1-x)}-1\right) \widehat{g}^{\delta}(\xi, \eta)$, equation (43) has a unique solution.

Lemma 3. If $\beta(x)$ is the solution of equation (43), then the following inequality holds

$$
\beta(x) \leq\left(\frac{(\tau-1) \delta}{E}\right)^{x-1}\left(\frac{1}{2} \ln \frac{E}{(\tau-1) \delta}\right)^{-p(1-x)}(1+o(1)) .
$$

Proof. Due to the triangle inequality and (43), note that $\left|T_{\beta}\right| \leq 1$, and we obtain

$$
\tau \delta=\left\|T_{\beta} \hat{g}^{\delta}\right\| \leq\left\|T_{\beta}\left(\hat{g}^{\delta}-\hat{g}\right)\right\|+\left\|T_{\beta} \hat{g} \leq \delta\right\|+\left\|T_{\beta} \hat{g}\right\| .
$$

We now estimate the second term on the right hand side of (46):

$$
\begin{aligned}
T_{\beta} \widehat{g} & =\left\|\left(e^{-(A+i B)(1-x)} k_{\beta}(x, \xi, \eta)-1\right) e^{-(A+i B)} \widehat{u}(0, \cdot, \cdot)\right\| \\
& =\left\|\frac{1-e^{-(A+i B)(1-x)} k_{\beta}(x, \xi, \eta)}{e^{A+i B}\left(1+\xi^{2}+\eta^{2}\right)^{p / 2}}\left(1+\xi^{2}+\eta^{2}\right)^{p / 2} \widehat{u}(0, \cdot, \cdot)\right\| \\
& \leq E \sup _{\xi, \eta \in \mathbb{R}, e^{A(1-x)}>\beta(x)}\left|\frac{1-e^{-A(1-x)} \beta(x)}{e^{A}\left(1+\xi^{2}+\eta^{2}\right)^{p / 2}}\right| \\
& =E \sup _{\xi, \eta \in \mathbb{R}, e^{A(1-x)}>\beta(x)}\left|\frac{e^{A(1-x)}-\beta(x)}{e^{A(2-x)}\left(1+\xi^{2}+\eta^{2}\right)^{p / 2}}\right| \\
& =E \sup _{\xi, \eta \in \mathbb{R}, e^{A(1-x)}>\beta(x)} \frac{e^{A(1-x)}-\beta(x)}{e^{A(1-x)}} e^{-A}\left(1+\xi^{2}+\eta^{2}\right)^{-(p / 2)} \\
& \leq E \sup _{\xi, \eta \in \mathbb{R}, e^{A(1-x)}>\beta(x)} e^{-A}\left(1+\xi^{2}+\eta^{2}\right)^{-(p / 2)} .
\end{aligned}
$$

Similar to the proof of Theorem 2, we can know

$$
\begin{aligned}
\left\|T_{\beta} \hat{g}\right\| \leq & E \sup _{\xi, \eta \in \mathbb{R}, e^{A(1-x)}>\beta(x)} e^{-A}\left(1+\xi^{2}+\eta^{2}\right)^{-(p / 2)} \\
\leq & E \beta(x)^{-(1 /(1-x))} \max \left\{\left(\frac{1}{2(1-x)} \ln \beta(x)\right)^{-p},\right. \\
& \left.\left(\frac{1}{2(1-x)} \ln \beta(x)\right)^{-((2 p) / \alpha)}\right\} \\
= & E \beta(x)^{-(1 /(1-x))}\left(\frac{1}{2(1-x)} \ln \beta(x)\right)^{-p} .
\end{aligned}
$$

Combining (46) and (48), we obtain

$$
\begin{aligned}
& (\tau-1) \delta \leq E \beta(x)^{-(1 /(1-x))}\left(\frac{1}{2(1-x)} \ln \beta(x)\right)^{-p} \cdot \quad \text { (49) } \\
& \text { Let } \quad(\tau-1) \delta=E \beta_{*}(x)^{-(1 /(1-x))}\left((1 /(2(1-x))) \ln \beta_{*}\right.
\end{aligned}
$$
$(x))^{-p}$. From Lemma 1 , we have

$$
\beta_{*}(x)=\left(\frac{(\tau-1) \delta}{E}\right)^{x-1}\left(\frac{1}{2} \ln \frac{E}{(\tau-1) \delta}\right)^{-p(1-x)}(1+o(1)) .
$$


Let $G(\beta):=E \beta(x)^{-(1 /(1-x))}((1 /(2(1-x))) \ln \beta(x))^{-p}$. For $\beta>1$ and $0 \leq x<1$, the function $G(\beta)$ is strictly decreasing monotonically with $\beta$, so we have

$$
\beta(x) \leq \beta_{*}(x) .
$$

Lemma 4. If $\beta(x)$ is the solution of equation (43), then the following inequality holds:

$$
\left\|T_{\beta} \hat{g}\right\| \leq(\tau+1) \delta .
$$

Proof. Using the triangle inequality and (43), we have

$$
\begin{aligned}
\left\|T_{\beta} \widehat{g}\right\| & =\left\|T_{\beta}\left(\widehat{g}-\widehat{g}^{\delta}\right)+T_{\beta} \widehat{\widehat{g}}^{\delta}\right\| \leq\left\|T_{\beta}\left(\widehat{g}-\widehat{g}^{\delta}\right)\right\|+\left\|T_{\beta} \widehat{g}^{\delta}\right\| \\
& \leq(\tau+1) \delta .
\end{aligned}
$$

Theorem 3. Assume that conditions (9) and (17) hold and taking the solution of equation (43) as the regularization parameter, then there holds the following error estimate:

$$
\left\|u_{\beta}^{\delta}(x, \cdot, \cdot)-u(x, \cdot, \cdot)\right\| \leq \delta^{x} E^{1-x}\left(\frac{1}{2} \ln \frac{E}{(\tau-1) \delta}\right)^{-p(1-x)}(C+o(1)),
$$

where $C=(\tau-1)^{x-1}(2 \tau+1), 0 \leq x<1$.

Proof. Due to the Parseval formula and the triangle inequality, we obtain

$$
\begin{aligned}
\left\|u_{\beta}^{\delta}(x, \cdot, \cdot)-u(x, \cdot, \cdot)\right\| & =\left\|\widehat{u}_{\beta}^{\delta}(x, \cdot, \cdot)-\widehat{u}(x, \cdot, \cdot)\right\| \\
& =\left\|k_{\beta} \widehat{g}^{\delta}-e^{(A+i B)(1-x)} \widehat{g}\right\| \\
& \leq\left\|k_{\beta}\left(\widehat{g}^{\delta}-\widehat{g}\right)\right\|+\left\|\left(k_{\beta}-e^{(A+i B)(1-x)}\right) \hat{g}\right\| \\
& \leq \beta(x) \delta+\left\|\left(k_{\beta} e^{-(A+i B)(1-x)}-1\right) \widehat{u}(x, \cdot, \cdot)\right\| \\
& =\beta(x) \delta+\left\|T_{\beta} \widehat{u}(x, \cdot, \cdot)\right\| .
\end{aligned}
$$

From Lemma 3, we can know that

$$
\beta(x) \delta \leq\left(\frac{E}{\tau-1}\right)^{1-x} \delta^{x}\left(\frac{1}{2} \ln \frac{E}{(\tau-1) \delta}\right)^{-p(1-x)}(1+o(1)) .
$$

In the following, we estimate the second term of (55). Denote $\varphi=T_{\beta} \widehat{u}(x, \cdot, \cdot)$ and take $\beta(x)=(E /((\tau-1) \delta))^{1-x}$ $((1 / 2) \ln (E /((\tau-1) \delta)))^{-p(1-x)}$; we have

$$
\begin{aligned}
\|\varphi\| & =\left\|\varphi-k_{\beta} e^{-(A+i B)(1-x)} \varphi+k_{\beta} e^{-(A+i B)(1-x)} \varphi\right\| \\
& \leq\left\|\varphi-k_{\beta} e^{-(A+i B)(1-x)} \varphi\right\|+\left\|k_{\beta} e^{-(A+i B)(1-x)} \varphi\right\| \\
& =M_{1}+M_{2} .
\end{aligned}
$$

Note that $\widehat{u}(x, \cdot, \cdot)=e^{(A+i B)(1-x)} \widehat{g}(\cdot, \cdot)$, we get

$$
\begin{aligned}
M_{1} & =\left\|T_{\beta} e^{(A+i B)(1-x)} \hat{g}\left(1-k_{\beta} e^{-(A+i B)(1-x)}\right)\right\| \\
& =\left\|T_{\beta}\left(e^{(A+i B)(1-x)}-k_{\beta}\right) \hat{g}\right\| \\
& \leq\left\|\left(e^{(A+i B)(1-x)}-k_{\beta}\right) \hat{g}\right\| .
\end{aligned}
$$

Denote $M:=\left\|\left(e^{(A+i B)(1-x)}-k_{\beta}\right) \hat{g}\right\|$, we have

$M=\left\|\left(e^{(A+i B)(1-x)}-k_{\beta}\right) \hat{g}\right\| \leq E \sup _{\xi, \eta \in \mathbb{R}}\left|\frac{e^{(A+i B)(1-x)}-k_{\beta}}{e^{(A+i B)}\left(1+\xi^{2}+\eta^{2}\right)^{p / 2}}\right|$

$$
\begin{aligned}
& \leq E \sup _{\xi, \eta \in \mathbb{R}}\left|\frac{e^{(A+i B)(1-x)}-\min \left\{e^{A(1-x)}, \beta(x)\right\} e^{i B(1-x)}}{e^{(A+i B)}\left(1+\xi^{2}+\eta^{2}\right)^{p / 2}}\right| \\
& \leq E \sup _{\xi, \eta \in \mathbb{R}, e^{A(1-x)}>\beta(x)} \frac{e^{A(1-x)}-\beta(x)}{e^{A}\left(1+\xi^{2}+\eta^{2}\right)^{p / 2}} \\
& =E \sup _{\xi, \eta \in \mathbb{R}, e^{A(1-x)}>\beta(x)} \frac{e^{A(1-x)}-\beta(x)}{e^{A(1-x)}}\left(1+\xi^{2}+\eta^{2}\right)^{-(p / 2)} e^{-A x} \\
& \leq E \sup _{\xi, \eta \in \mathbb{R}, e^{A(1-x)}>\beta(x)}\left(1+\xi^{2}+\eta^{2}\right)^{-(p / 2)} e^{-A x} .
\end{aligned}
$$

Similar to the proof of Theorem 2, we can know that 


$$
\begin{aligned}
& M \leq E \sup _{\xi, \eta \in \mathbb{R}, e^{A(1-x)}>\beta(x)}\left(1+\xi^{2}+\eta^{2}\right)^{-(p / 2)} e^{-A x} \\
& \leq E \beta(x)^{-(x /(1-x))} \max \left\{\left(\frac{1}{2(1-x)} \ln \beta(x)\right)^{-p},\left(\frac{1}{2(1-x)} \ln \beta(x)\right)^{-((2 p) / \alpha)}\right\} \\
& =E \beta(x)^{-(x /(1-x))}\left(\frac{1}{2(1-x)} \ln \beta(x)\right)^{-p} \\
& =E\left[\frac{1}{2(1-x)} \ln \left(\left(\frac{E}{(\tau-1) \delta}\right)^{1-x}\left(\frac{1}{2} \ln \frac{E}{(\tau-1) \delta}\right)^{-p(1-x)}\right)\right]^{-p} \\
& \times\left[\left(\frac{E}{(\tau-1) \delta}\right)^{1-x}\left(\frac{1}{2} \ln \frac{E}{(\tau-1) \delta}\right)^{-p(1-x)}\right]^{-(x /(1-x))} \\
& =E\left[\frac{1}{2} \ln \frac{E}{(\tau-1) \delta}+\ln \left(\frac{1}{2} \ln \frac{E}{(\tau-1) \delta}\right)^{-(p / 2)}\right]^{-p}\left(\frac{E}{(\tau-1) \delta}\right)^{-x}\left(\frac{1}{2} \ln \frac{E}{(\tau-1) \delta}\right)^{p x} \\
& =E\left(\frac{1}{2} \ln \frac{E}{(\tau-1) \delta}\right)^{-p}\left[1+\frac{\ln (1 / 2 \ln E /(\tau-1) \delta)^{-(p / 2)}}{1 / 2 \ln E /(\tau-1) \delta}\right]^{-p}\left(\frac{(\tau-1) \delta}{E}\right)^{x}\left(\frac{1}{2} \ln \frac{E}{(\tau-1) \delta}\right)^{p x} \\
& =(\tau-1)^{x} \delta^{x} E^{1-x}\left(\frac{1}{2} \ln \frac{E}{(\tau-1) \delta}\right)^{-p(1-x)}(1+o(1)) \text {. } \\
& g(y, t)=\left(g\left(y_{1}, t_{1}\right), g\left(y_{2}, t_{2}\right), \ldots, g\left(y_{n}, t_{n}\right)\right)^{T}, \\
& y_{i}=(i-1) \Delta y, \quad \Delta y=\frac{1}{n-1}, \quad i=1,2, \ldots, n, \\
& t_{j}=(j-1) \Delta t, \quad \Delta t=\frac{1}{n-1}, \quad j=1,2, \ldots, n,
\end{aligned}
$$

For $M_{2}$, from Lemma 4 and assuming that $\left|k_{\beta}\right| \leq \beta(x)$, we have

$$
\begin{aligned}
M_{2} & \leq \beta(x)\left\|e^{-(A+i B)(1-x)} \varphi\right\| \\
& =\beta(x)\left\|T_{\beta} \hat{g}\right\| \\
& \leq(\tau+1)(\tau-1)^{x-1} \delta^{x} E^{1-x}\left(\frac{1}{2} \ln \frac{E}{(\tau-1) \delta}\right)^{-p(1-x)} .
\end{aligned}
$$

Combining (56)-(61) with (55), the proof of Theorem 3 is completed.

\section{Numerical Aspect}

In this section, we present two numerical examples intended to illustrate the behavior of the proposed method.

The numerical examples are constructed in the following way. First, we present the initial data $u(0, y, t)=f(y, t)$ of $2 \mathrm{D}$ time-fractional diffusion problem at $x=0$ and computed the function $u(1, y, t)=g(y, t)$ by solving a direct problem, which is a well-posed problem. Then, we added a random distributed perturbation to the data function obtaining vector $g^{\delta}(y, t)$, i.e.,

$$
g^{\delta}(y, t)=g(y, t)+\varepsilon \operatorname{randn}(\operatorname{size}(g(y, t))),
$$

where where $\delta$ indicates the error level of $g$, i.e.,

$$
\delta:=\sqrt{\frac{1}{n^{2}} \sum_{i=1}^{n} \sum_{j=1}^{n}\left(g^{\delta}\left(y_{i}, t_{j}\right)-g\left(y_{i}, t_{j}\right)\right)^{2}} .
$$

In numerical implementations, we give the data $f(y, t)$ and sample at an equidistant grid in the domain $[0,1] \times[0,1]$ with $64 \times 64$ grid points. The function "randn $(\cdot)$ " generates arrays of random numbers whose elements are normally distributed with mean 0 , variance $\sigma^{2}=1$, and standard deviation $\sigma=1$; "randn size $(g(y, t))$ " returns an array of random entries which has the same size as $g(y, t)$. Let RMS denotes the mean square for a sampled function $\varphi(\cdot, \cdot)$ which is defined by

$$
\operatorname{RMS}(\varphi):=\sqrt{\frac{1}{n^{2}} \sum_{i=1}^{n} \sum_{j=1}^{n}\left(\varphi\left(y_{i}, t_{j}\right)\right)^{2}},
$$

where $n$ is the total number of test points. Similarly, we can define the mean square error (RMSE) between the computed data and the exact data.

Finally, we solved the 2D time-fractional inverse diffusion problem by the modified method.

Example 1. We consider a smooth function 


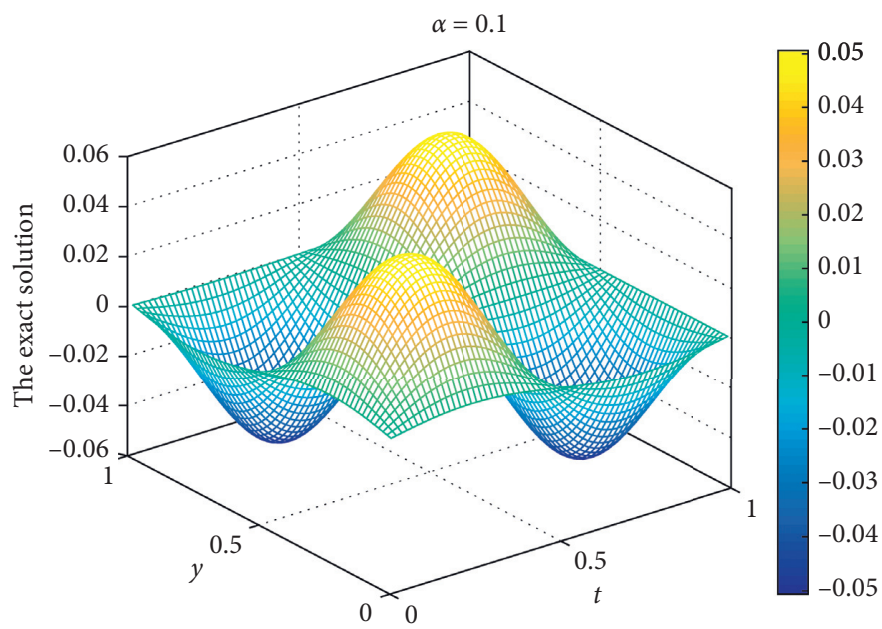

(a)

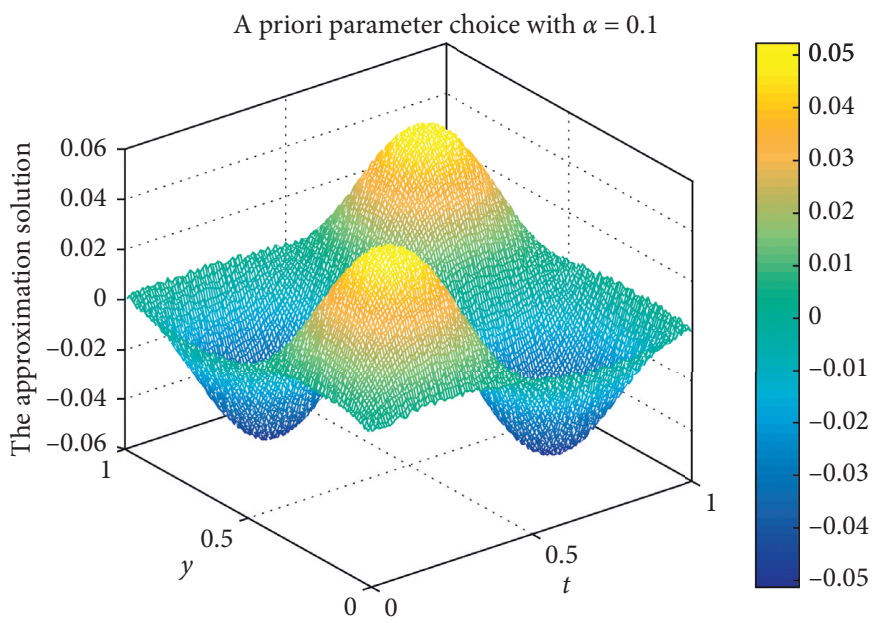

(b)

Figure 1: The exact solution and its approximation solution at $\varepsilon=0.001$ and $\alpha=0.1$ : (a) the exact solution at $x=0.9$; (b) its approximation solution at $x=0.9$.

$$
u(0, y, t)=f(y, t)=16 \sin (2 \pi y) \sin (2 \pi t)
$$

Example 2. Let $\Omega=\{(y, t) \quad \mid 0.1 \leq y \leq 0.3,0.1 \leq t \leq 0.3\} \cup$ $\{(y, t) \mid 0.6 \leq y \leq 0.8,0.6 \leq t \leq 0.8\}$. Consider a nonsmooth function

$$
f(y, t)= \begin{cases}1, & \text { if }(\mathrm{y}, \mathrm{t}) \in \Omega, \\ 0, & \text { if else. }\end{cases}
$$

Figures 1-3 are the comparison of the exact solution and the approximation solution for $\alpha=0.1$ with noise level $\varepsilon=$ 0.001 at different points $x=0.9,0.5$, and 0.1 under a priori regularization parameter choice rule with Example 1. Figures 4-6 are the comparison of the exact solution and the approximation solution for $\alpha=0.1$ with noise level $\varepsilon=0.0001$ at different points $x=0.9,0.5$, and0.1 under a posteriori regularization parameter choice rule with Example 1 .

Table 1 shows the comparison of RMSE of Example 1 for different $x$ with $\varepsilon=0.0001$ and $\alpha=0.1$. We find that the smaller the $x$ is, the worse the computed approximation will be.

Table 2 shows the comparison of RMSE of Example 1 for different $\varepsilon$ with $\alpha=0.1$ at $x=0.5$. The numerical error is decreasing as the level of noise becomes smaller.

Table 3 shows the comparison of RMSE of Example 1 for different $\alpha$ with $\varepsilon=0.0001$ at $x=0.5$. The numerical accuracy is stable to the fractional order $\alpha$.

Figure 7 is the comparison of the exact solution and the approximation solution for $\alpha=0.1$ with noise level $\varepsilon=0.0001$ at $x=0.4$ under both a priori and a posteriori regularization parameter choice rule with Example 2. 


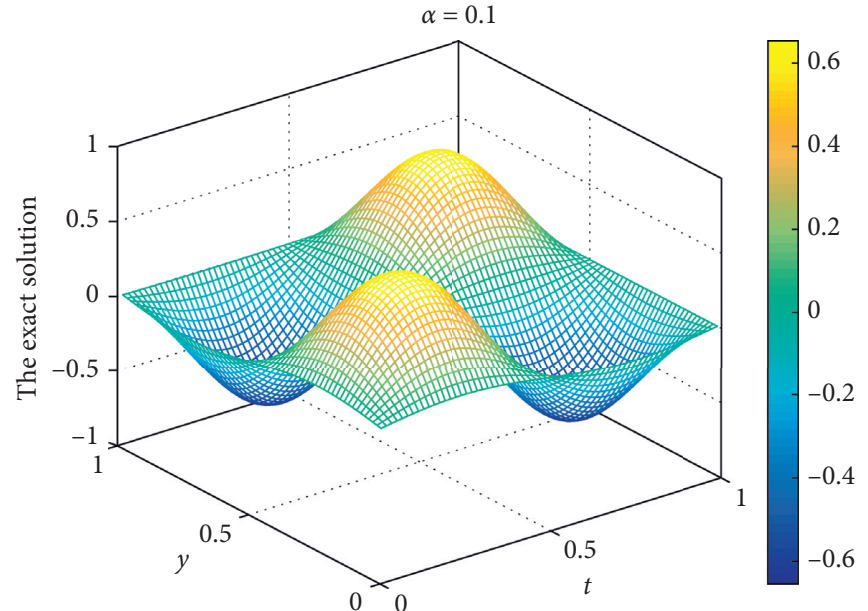

(a)

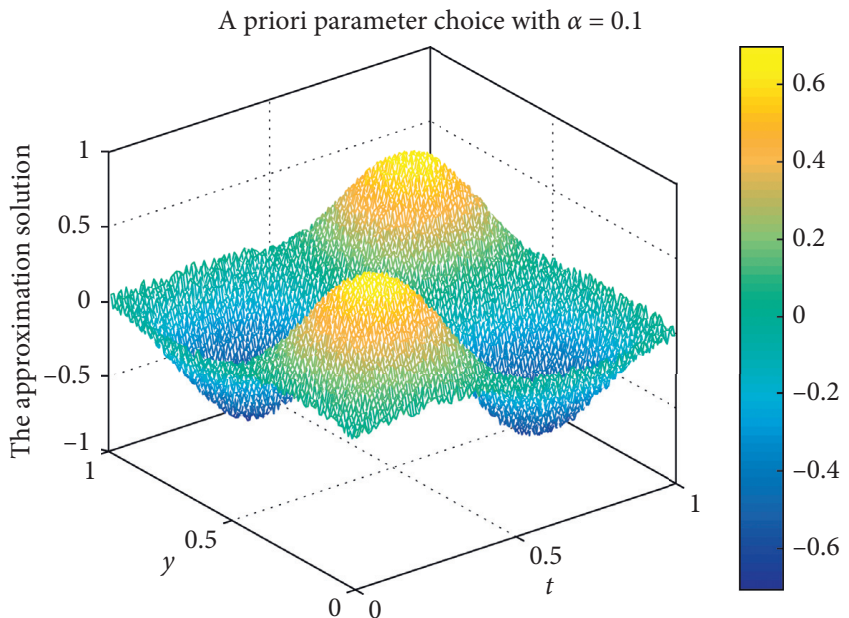

(b)

FIgURE 2: The exact solution and its approximation solution at $\varepsilon=0.001$ and $\alpha=0.1$ : (a) the exact solution at $x=0.5$; (b) its approximation solution at $x=0.5$.

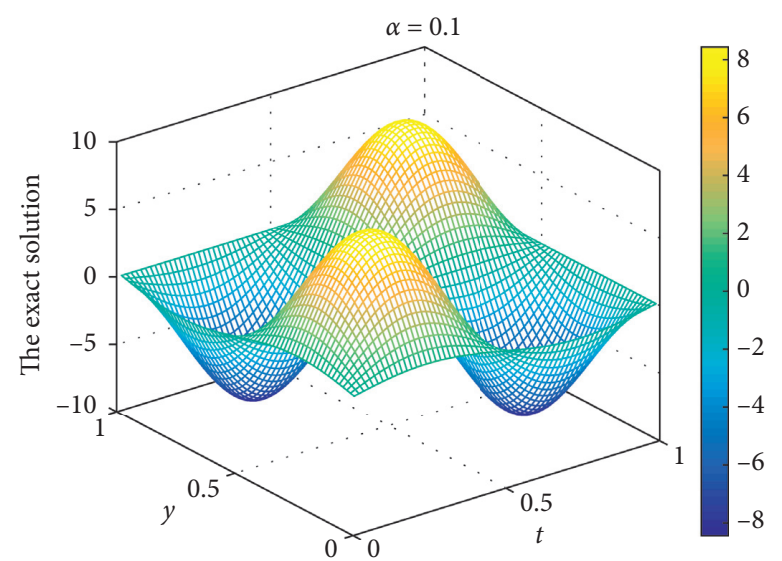

(a)

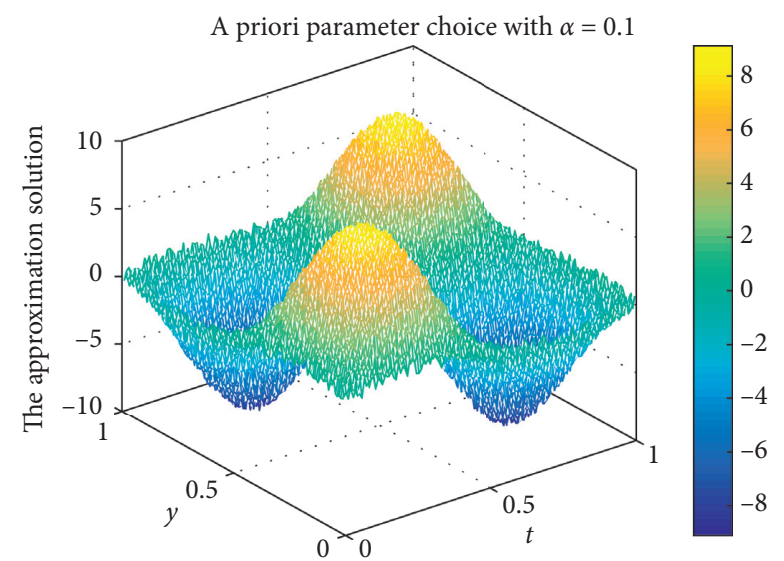

(b)

FIgURE 3: The exact solution and its approximation solution at $\varepsilon=0.001$ and $\alpha=0.1$ : (a) the exact solution at $x=0.1$; (b) its approximation solution at $x=0.1$.

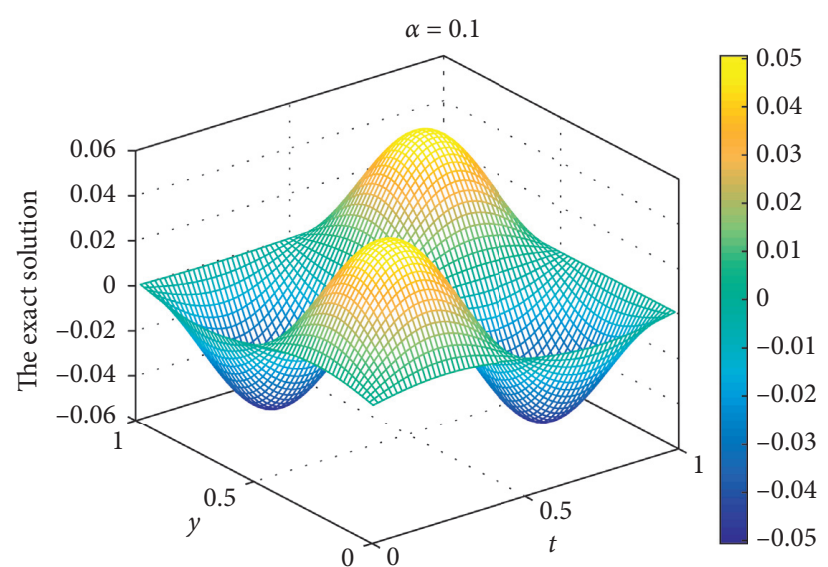

(a)

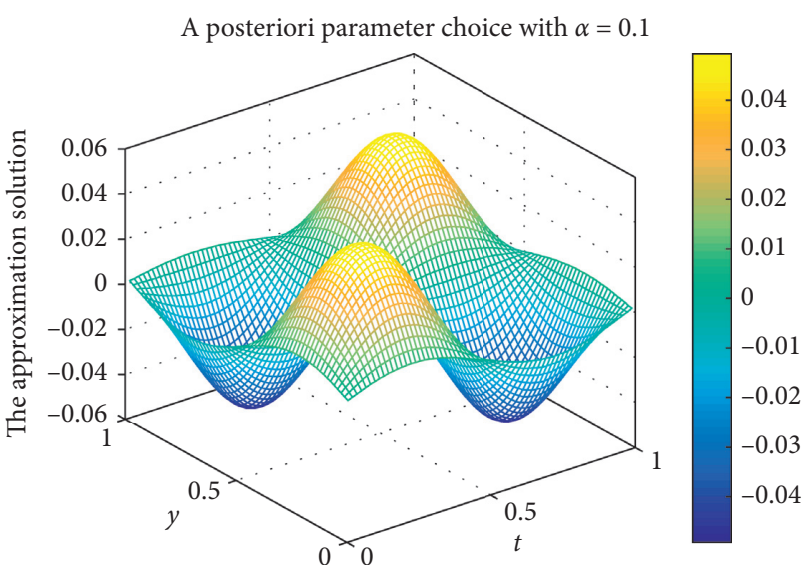

(b)

FIgURE 4: The exact solution and its approximation solution at $\varepsilon=0.0001$ and $\alpha=0.1$ : (a) the exact solution at $x=0.9$; (b) its approximation solution at $x=0.9$. 


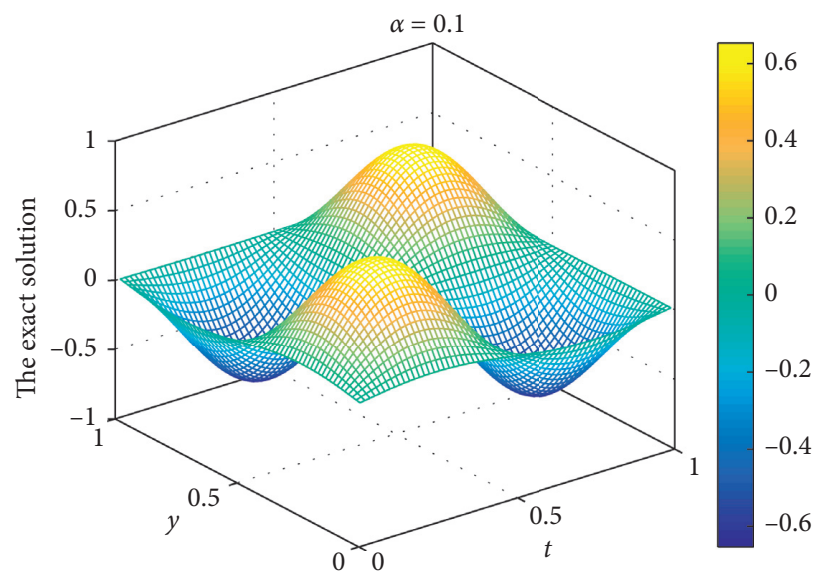

(a)

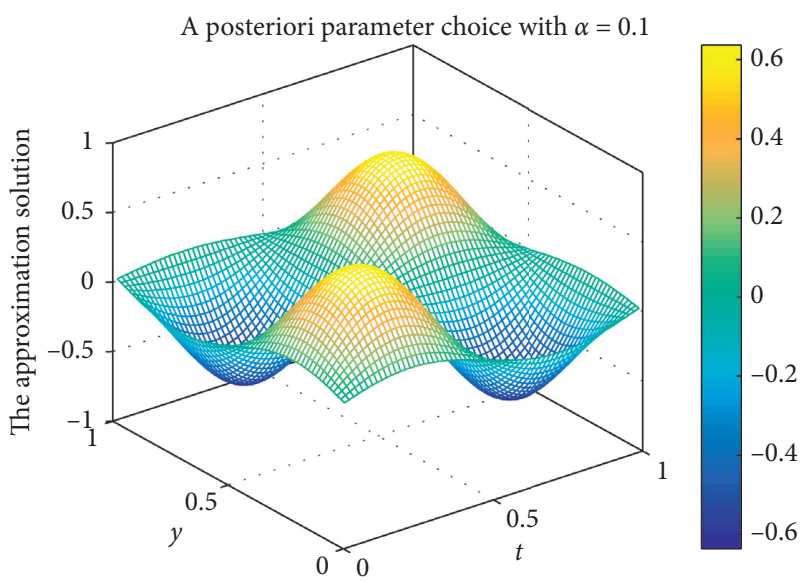

(b)

FIgURE 5: The exact solution and its approximation solution at $\varepsilon=0.0001$ and $\alpha=0.1$ : (a) the exact solution at $x=0.5$; (b) its approximation solution at $x=0.5$.

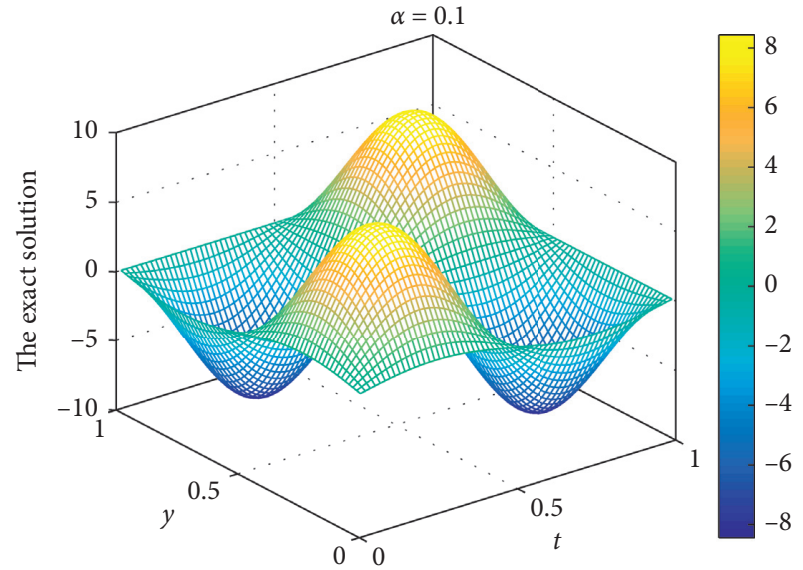

(a)

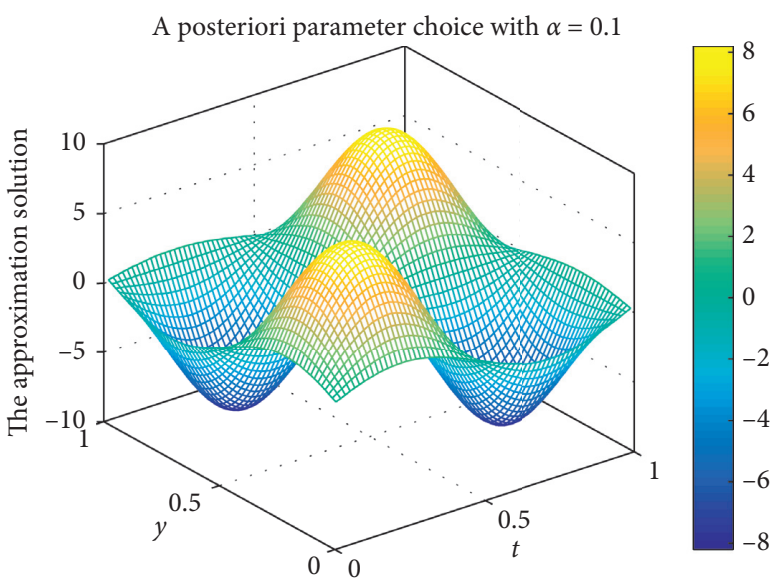

(b)

Figure 6: The exact solution and its approximation solution at $\varepsilon=0.0001$ and $\alpha=0.1$ : (a) the exact solution at $x=0.1$; (b) its approximation solution at $x=0.1$.

TABLE 1: Error behavior of Example 1 for different $x$ with $\alpha=0.1$ and $\varepsilon=0.0001$.

\begin{tabular}{lccc}
\hline$x$ & 0.5 & 0.3 & 0.1 \\
\hline A priori choice rule: RMSE & $4.59 \times 10^{-2}$ & $1.73 \times 10^{-1}$ & $6.37 \times 10^{-1}$ \\
A posteriori choice rule: RMSE & $4.55 \times 10^{-2}$ & $1.63 \times 10^{-1}$ & $5.88 \times 10^{-1}$ \\
\hline
\end{tabular}

TABLE 2: Error behavior of Example 1 for different $\varepsilon$ with $\alpha=0.1$ at $x=0.5$.

\begin{tabular}{lccc}
\hline$\varepsilon$ & 0.01 & 0.001 & 0.0001 \\
\hline A priori choice rule: RMSE & $9.22 \times 10^{-2}$ & $5.16 \times 10^{-2}$ & $4.59 \times 10^{-2}$ \\
A posteriori choice rule: RMSE & $8.94 \times 10^{-2}$ & $4.90 \times 10^{-2}$ & $4.55 \times 10^{-2}$ \\
\hline
\end{tabular}

TABle 3: Error behavior of Example 1 for different $\alpha$ with $\varepsilon=0.0001$ at $x=0.5$.

\begin{tabular}{lccc}
\hline$\alpha$ & 0.1 & 0.3 & 0.8 \\
\hline A priori choice rule: RMSE & $4.59 \times 10^{-2}$ & $4.38 \times 10^{-2}$ & $3.89 \times 10^{-2}$ \\
A posteriori choice rule: RMSE & $4.55 \times 10^{-2}$ & $4.35 \times 10^{-2}$ & $3.88 \times 10^{-2}$ \\
\hline
\end{tabular}




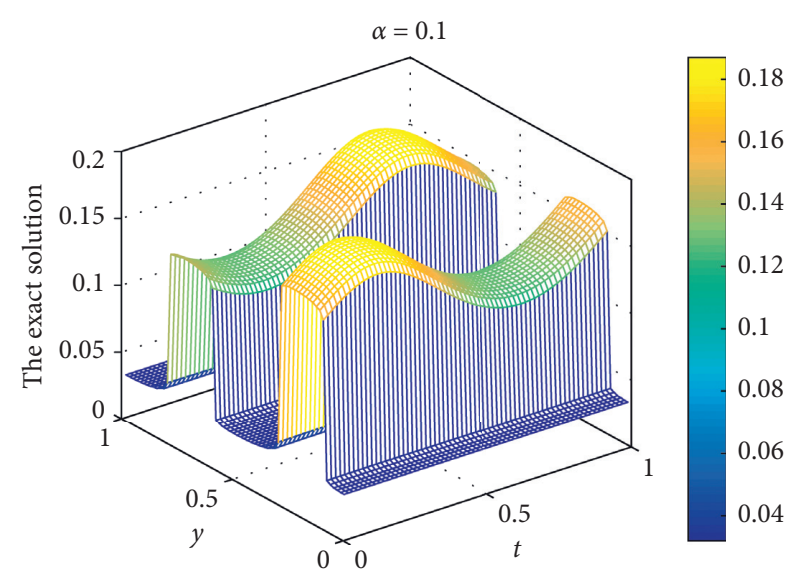

(a)

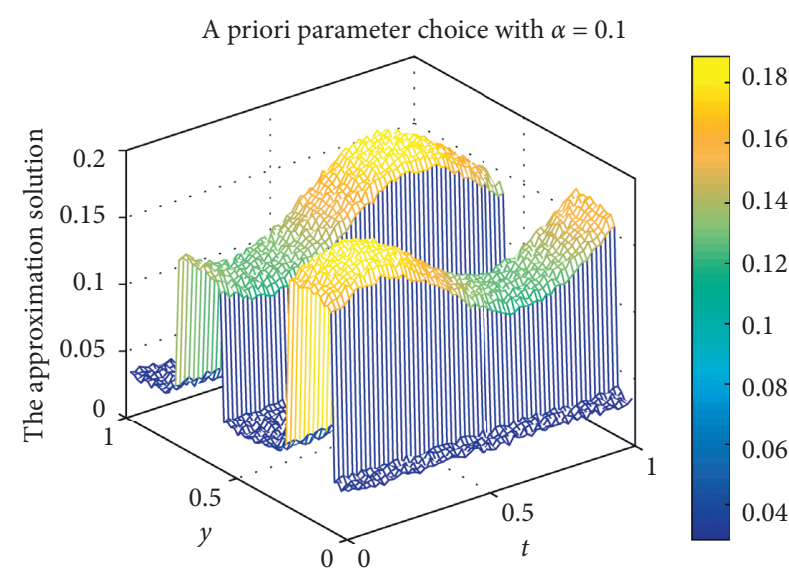

(b)

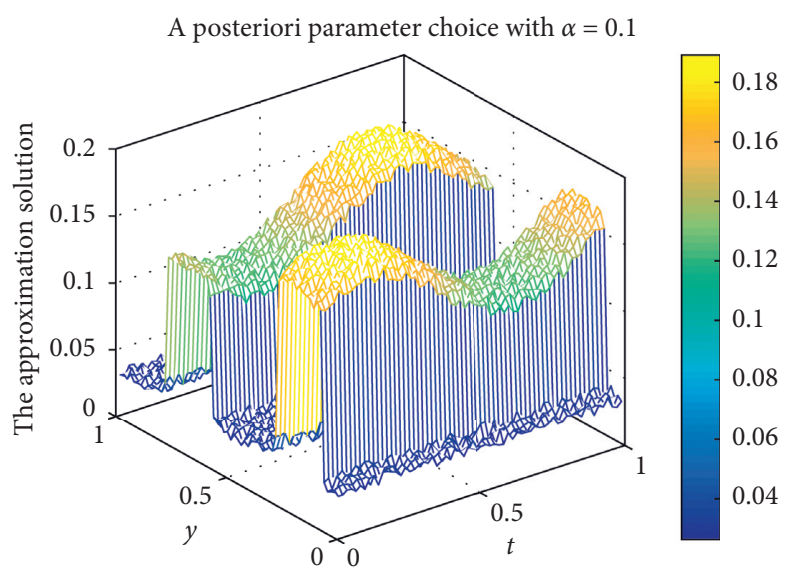

(c)

FiguRE 7: The exact solution and its approximation solution at $\varepsilon=0.0001$ and $\alpha=0.1$ : (a) the exact solution at $x=0.4$; (b) its approximation solution under a priori parameter choice rule at $x=0.4$; (c) its approximation solution under a posteriori parameter choice rule at $x=0.4$.

\section{Conclusion}

In this paper, we propose a modified method to solve the time-fractional inverse diffusion problem in the two-dimensional setting. For the choice of regularization parameter, we give not only a priori but also a posteriori rules. Moreover, under both a priori rule and a posteriori rule, we prove the error estimates from the viewpoint of theoretical analysis.

The numerical examples are presented to illustrate the validity and effectiveness of the proposed method. In general, $E>0$ such that a priori condition (17) holds will not be known. But such a $E$ has to be known if one wants to construct a priori parameter choice rule. We propose a posteriori parameter choice rule which is independent of $E$. So it is more implementable in practical application. Moreover, the numerical experiments show that a posteriori parameter choice rule also works well.

\section{Data Availability}

The data used to support the findings of this study are available from the corresponding author upon request.

\section{Conflicts of Interest}

The authors declare that they have no conflicts of interest.

\section{Authors' Contributions}

All authors contributed equally to the writing of this paper. All authors read and approved the final manuscript.

\section{Acknowledgments}

This work was partially supported by the National Natural Science Foundation of China (11871198), the Fundamental Research Funds for the Universities of Heilongjiang Province Heilongjiang University Special Project (RCYJTD201804), the National Science Foundation of Hebei Province (A2017501021), and the Fundamental Research Funds of the Central Universities (N182304024).

\section{References}

[1] W. Chen, L. Ye, and H. Sun, "Fractional diffusion equations by the Kansa method," Computers \& Mathematics with Applications, vol. 59, no. 5, pp. 1614-1620, 2010. 
[2] Z. Yu and J. Lin, "Numerical research on the coherent structure in the viscoelastic second-order mixing layers," Applied Mathematics and Mechanics, vol. 19, no. 8, pp. 717723, 1998.

[3] H. Scher and E. W. Montroll, "Anomalous transit-time dispersion in amorphous solids," Physical Review B, vol. 12, no. 6, pp. 2455-2477, 1975.

[4] T. L. Szabo and J. Wu, "A model for longitudinal and shear wave propagation in viscoelastic media," The Journal of the Acoustical Society of America, vol. 107, no. 5, pp. 2437-2446, 2000.

[5] R. Gorenflo, F. Mainardi, D. Moretti, G. Pagnini, and P. Paradisi, "Discrete random walk models for space-time fractional diffusion," Chemical Physics, vol. 284, no. 1-2, pp. 521-541, 2002.

[6] C. Wang, L. Ling, X. Xiong, and M. Li, "Regularization for 2-D fractional sideways heat equations," Numerical Heat Transfer, Part B: Fundamentals, vol. 68, no. 5, pp. 418-433, 2015.

[7] R. V. Mendes, "A fractional calculus interpretation of the fractional volatility model," Nonlinear Dynamics, vol. 55, no. 4, pp. 395-399, 2009.

[8] B. Berkowitz, H. Scher, and S. E. Silliman, "Anomalous transport in laboratory-scale, heterogeneous porous media," Water Resources Research, vol. 36, no. 1, pp. 149-158, 2000.

[9] R. Metzler and J. Klafter, "The random walk's guide to anomalous diffusion: a fractional dynamics approach," Physics Reports, vol. 339, no. 1, pp. 1-77, 2000.

[10] E. Scalas, R. Gorenflo, and F. Mainardi, "Fractional calculus and continuous-time finance," Physica A: Statistical Mechanics and Its Applications, vol. 284, no. 1-4, pp. 376-384, 2000.

[11] I. M. Sokolov and J. Klafter, "From diffusion to anomalous diffusion: a century after Einsteins Brownian motion," Chaos: An Interdisciplinary Journal of Nonlinear Science, vol. 15, no. 2, Article ID 026103, 2005.

[12] L. R. Evangelista and E. K. Lenzi, Fractional Diffusion Equations and Anomalous Diffusion, Cambridge University Press, Cambridge, UK, 2018.

[13] A. A. Tateishi, H. V. Ribeiro, and E. K. Lenzi, "The role of fractional time-derivatives operators on anomalous diffusion," Frontiers in Physics, vol. 5, p. 52, 2017.

[14] S. D. Eidelman and A. N. Kochubei, "Cauchy problem for fractional diffusion equations," Journal of Differential Equations, vol. 199, no. 2, pp. 211-255, 2004.

[15] R. Gorenflo, Y. F. Luchko, and P. P. Zabrejko, "On solvability of linear fractional differential equations in Banach spaces," Fractional Calculus and Applied Analysis, vol. 2, pp. 163-176, 1999.

[16] A. Hanyga, "Multidimensional solutions of time-fractional diffusion-wave equations," Proceedings of the Royal Society of London. Series A: Mathematical, Physical and Engineering Sciences, vol. 458, no. 2018, pp. 933-957, 2002.

[17] Y. Luchko, "Maximum principle for the generalized timefractional diffusion equation," Journal of Mathematical Analysis and Applications, vol. 351, no. 1, pp. 218-223, 2009.

[18] Y. Luchko, "Some uniqueness and existence results for the initial-boundary-value problems for the generalized timefractional diffusion equation," Computers \& Mathematics with Applications, vol. 59, no. 5, pp. 1766-1772, 2010.

[19] F. Liu, V. Anh, and I. Turner, "Numerical solution of the space fractional Fokker-Planck equation," Journal of Computational and Applied Mathematics, vol. 166, no. 1, pp. 209-219, 2004.

[20] F. Liu, P. Zhuang, V. Anh, I. Turner, and K. Burrage, "Stability and convergence of the difference methods for the space-time fractional advection-diffusion equation," Applied Mathematics and Computation, vol. 191, no. 1, pp. 12-20, 2007.

[21] M. M. Meerschaert and C. Tadjeran, "Finite difference approximations for fractional advection-dispersion flow equations," Journal of Computational and Applied Mathematics, vol. 172, no. 1, pp. 65-77, 2004.

[22] M. M. Meerschaert, Y. Zhang, and B. Baeumer, "Particle tracking for fractional diffusion with two time scales," Computers \& Mathematics with Applications, vol. 59, no. 3, pp. 1078-1086, 2010.

[23] Q. Yang, F. Liu, and I. Turner, "Numerical methods for fractional partial differential equations with Riesz space fractional derivatives," Applied Mathematical Modelling, vol. 34, no. 1, pp. 200-218, 2010.

[24] Z. Yang, Y. Nie, Z. Yuan, and J. Wang, "Finite element methods for fractional PDEs in three dimensions," Applied Mathematics Letters, vol. 100, Article ID 106041, 2020.

[25] D. A. Murio, "Stable numerical solution of a fractional-diffusion inverse heat conduction problem," Computers \& Mathematics with Applications, vol. 53, no. 10, pp. 1492-1501, 2007.

[26] D. A. Murio, "Time fractional IHCP with Caputo fractional derivatives," Computers \& Mathematics with Applications, vol. 56, no. 9, pp. 2371-2381, 2008.

[27] J. Cheng, J. Nakagawa, M. Yamamoto, and T. Yamazaki, "Uniqueness in an inverse problem for a one-dimensional fractional diffusion equation," Inverse Problems, vol. 25, no. 11, Article ID 115002, 2009.

[28] J. J. Liu and M. Yamamoto, "A backward problem for the time-fractional diffusion equation," Applicable Analysis, vol. 89, no. 11, pp. 1769-1788, 2010.

[29] Y. Zhang and $\mathrm{X}$. Xu, "Inverse source problem for a fractional diffusion equation," Inverse Problems, vol. 27, no. 3, Article ID 035010, 2011.

[30] G. H. Zheng and T. Wei, "A new regularization method for solving a time-fractional inverse diffusion problem," Journal of Mathematical Analysis and Applications, vol. 378, no. 2, pp. 418-431, 2011.

[31] B. T. Jin and W. Rundell, "An inverse problem for a onedimensional time-fractional diffusion problem," Inverse Problems, vol. 28, no. 7, Article ID 075010, 2012.

[32] T. Wei and J. Wang, "A modified quasi-boundary value method for an inverse source problem of the time-fractional diffusion equation," Applied Numerical Mathematics, vol. 78, pp. 95-111, 2014.

[33] T. Wei, X. L. Li, and Y. S. Li, "An inverse time-dependent source problem for a time-fractional diffusion equation," Inverse Problems, vol. 32, no. 8, Article ID 085003, 2016.

[34] G. H. Zheng and T. Wei, "Spectral regularization method for solving a time-fractional inverse diffusion problem," Applied Mathematics and Computation, vol. 218, no. 2, pp. 396-405, 2011.

[35] H. Cheng and C.-L. Fu, "An iteration regularization for a time-fractional inverse diffusion problem," Applied Mathematical Modelling, vol. 36, no. 11, pp. 5642-5649, 2012.

[36] X. Xiong, H. Guo, and X. Liu, "An inverse problem for a fractional diffusion equation," Journal of Computational and Applied Mathematics, vol. 236, no. 17, pp. 4474-4484, 2012.

[37] M. Li, X.-X. Xi, and X.-T. Xiong, "Regularization for a fractional sideways heat equation," Journal of Computational and Applied Mathematics, vol. 255, pp. 28-43, 2014.

[38] X. Xiong, Q. Zhou, and Y. C. Hon, “An inverse problem for fractional diffusion equation in 2-dimensional case: stability analysis and regularization," Journal of Mathematical Analysis and Applications, vol. 393, no. 1, pp. 185-199, 2012. 
[39] I. Podlubny, Fractional Differential Equations, Academic Press, San Diego, CA, USA, 1999.

[40] U. Tautenhahn, "Optimality for ill-posed problems under general source conditions," Numerical Functional Analysis and Optimization, vol. 19, no. 3-4, pp. 377-398, 1998.

[41] A. Kirsch, An Introduction to the Mathematical Theory of Inverse Problem, Springer, New York, NY, USA, 1999. 